ESAIM: PROCEEDINGS, April 2009, Vol. 26, p. 123-134

H. Ammari, Editor

\title{
IMAGING OF A SMALL DIELECTRIC SPHERE BURIED IN A HALF SPACE
}

\author{
S. Gdoura ${ }^{1}$, D. Lesselier $^{1}$, P. C. Chaumet $^{2}$ and G. Perrusson ${ }^{1}$
}

\begin{abstract}
A MUSIC-type non-iterative, single-frequency imaging method is investigated, in line with recent works by the authors and colleagues on the subject. A small dielectric sphere is buried within the lower half of a two-half-space medium and illuminated from the upper one by a planar electric dipole array. Data of the imaging, the Multi-Static Response Matrix (MSR) involving the dyadic Green function of the medium and exploited via singular value decomposition, are either exact or asymptotic ones. Exact ones are computed by the Coupled Dipole Method (CDM). Asymptotic ones account for coupling to the interface via a Polarization Tensor (PT) calculated in closed form in the bispherical coordinate system attached to the (two-body) sphere-interface system. Comparisons between field data and between images of the inclusion inferred from them are discussed. Among other items, the capabilities of localization of the imaging procedure are exhibited. Whenever the inclusion is far enough from the interface, simplifying the PT to the one of an inclusion in free space is shown to be reliable. Use of a leading-order approximation of the Green function to speed up the imaging procedure is also investigated.
\end{abstract}

\section{INTRODUCTION}

MUSIC-type imaging of volumetric (3-D bounded) inclusions from single-frequency scattered field data (the MultiStatic Response or MSR matrix) is developed in a series of contributions by the authors and colleagues, [5], [15], [16]. Yet a number of questions remain if one carries it out for inclusions that are not so small with respect to the wavelength in the embedding medium, and/or not well separated from one another or from the boundaries of their embedding medium. Indeed, the processing of the MSR matrix is valid in theory if it is calculated from the first-order term of an asymptotic formulation constructed as a function of the volume of the inclusions, Polarization Tensors (PT) accounting for coupling between inclusions and/or between inclusions and boundaries being involved in that term ( [1]).

The case of large inclusions in free space is illustrated in [14] from data that are prone to inverse crime (calculated in the asymptotic framework) as well as from data provided by a full-field method which is involving no other errors than those due to discretization - the Coupled Dipole Method, [9]. Here, the work focuses onto a spherical inclusion which is buried in a half space, illuminated and seen from the other half space. In contrast with [15], the fact that this sphere might be found at shallow depth (a fraction of the wavelength, and down to a distance of the order of its radius) is accounted for in the asymptotic formulations when collecting the data. This involves a Polarization Tensor (PT) [13] which accounts for coupling of the inclusion to the interface (this is a two-body configuration), via closed forms developed within a bispherical system of coordinates attached to it, [7], [12]. That being done, one is able to study the effect of this coupling both on the MSR matrix and

\footnotetext{
${ }^{1}$ Département de Recherche en Électromagnétisme, Laboratoire des Signaux et Systèmes, UMR 8506 (CNRS-Supélec-Université Paris-Sud 11), Gif-sur-Yvette, France.

${ }^{2}$ Institut Fresnel, UMR 6133, Marseille, France.
}

(c) EDP Sciences, SMAI 2009 
the imaging, and to investigate the reliability of an imaging based on an asymptotic theory when true data are input, like those provided by the CDM. In addition, the Leading Order Approximation (LOA) of the Green dyad ( [11]) as used to speed up the imaging is investigated. Numerical simulations from CDM and asymptotic ones, with possibly strong noise, illustrate the above.

Many works dedicated to non-iterative imaging exists already, refer without pretense to exhaustivity to the references in the aforementioned MUSIC-type papers. Still, the problem at hand, which is a fully 3-D vector electromagnetic one, considered both in an asymptotic framework (via a two-body PT) and by exact means (by using CDM-computed data), is not attacked elsewhere to the best of our knowledge, effort in that direction for several inclusions (without interface) using the Lax-Foldy theory being noticed however [18]. In this connection, it is also worth mentioning the papers [3] and [6], where the PT of two nearly touching inclusions and the PT of an inclusion near a planar interface have been computed.

The present paper is organized as follows. In section 2, the direct problem is sketched. Emphasis is on the calculation of the PT of the two-body system. In section 3 the non-iterative imaging is considered, main elements of the algorithm being reminded with due reference to earlier works. In section 4, comparisons between numerical results obtained via the asymptotic method and via the CDM are proposed, preceded by examples of the influence of coupling to the interface on the behavior of the PT and consequences in terms of fields. A brief conclusion follows. The Appendix provides some necessary inforamtion about the bispherical system and so-called R-separability.

\section{Direct PRoblem}

Let us refer to Fig. 1. Two homogeneous dielectric half spaces (permeability $\mu_{0}$ ) with planar interface at $z=0$ are considered. The upper one is of permittivity $\varepsilon_{0}$ (air, typically); the lower one is of permittivity $\varepsilon_{-}$. A spherical dielectric inclusion with radius $a$ (volume denoted as $V$ ) and permittivity $\varepsilon_{\star}$ is buried in the lower half space at location $\mathbf{x}_{\star}$ (its center) $-d$ distance center-to-interface. It is illuminated by a planar source array, symmetric with respect to the $z$-axis, in the upper half space at distance $h$ from the interface; this

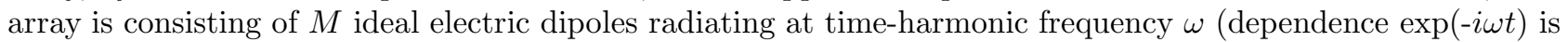
henceforth implied), all such dipoles orientated parallel to the vertical $z$-axis. This array is also operated as a receiver array in order to collect the vertical components of the scattered electric field when one given dipole (the same or any of the others in the array) is emitting, which is yielding the Multi-Static Response matrix (MSR) of the scatterer for the (single) $z$-polarization of transmitters and receivers.

The incident electric field radiated at arbitrary point $\mathbf{r}$ by the $n$th dipole of current $I_{n}$ and location $\mathbf{r}_{n}$ is given by

$$
\mathbf{E}_{0}^{(n)}(\mathbf{r})=i \omega \mu_{0} \underline{\mathbf{G}}^{e e}\left(\mathbf{r}, \mathbf{r}_{n}\right) \cdot \hat{z} I_{n} .
$$

where the Green tensor of the embedding two-half-space medium for an electric excitation, $\underline{\mathbf{G}}^{e e}$, reads as $\nabla \times \nabla \times \underline{\mathbf{G}}^{e e}\left(\mathbf{r}, \mathbf{r}^{\prime}\right)-\omega^{2} \varepsilon(\mathbf{r}) \mu_{0} \underline{\mathbf{G}}^{e e}\left(\mathbf{r}, \mathbf{r}^{\prime}\right)=\underline{\mathbf{I}} \delta\left(\mathbf{r}-\mathbf{r}^{\prime}\right)$, $\underline{\mathbf{I}}$ being the $3 \times 3$-identity matrix, and $\varepsilon(\mathbf{r})$ either $\varepsilon_{0}$ or $\varepsilon_{-}$ depending upon $\mathbf{r}$. In addition, transmission boundary conditions at the interface and far-field one at infinity apply. As for the reciprocity theorem, it yields $\underline{\mathbf{G}}^{e e}\left(\mathbf{r}, \mathbf{r}^{\prime}\right)=\left[\underline{\mathbf{G}}^{e e}\left(\mathbf{r}^{\prime}, \mathbf{r}\right)\right]^{t}$, where the upper index $t$ denotes transposition.

\subsection{The asymptotic formula of the scattered field}

The derivation being skipped for brevity (one should refer to the aforementioned references for most details), from the Lippmann-Schwinger domain integral formulation satisfied by the field, one builds up an asymptotic series expansion of the scattered electric field everywhere in space versus positive integer powers $(3,5$, then higher) of the spherical inclusion radius $a$. The lowest-order term is expected to accurately reproduce the true field if $a$ is much smaller than the wavelength in the embedding medium. Here, we have

$$
\mathbf{E}_{s}^{(n)}(\mathbf{r})=\frac{\mu_{0}}{i \omega} \underline{\mathbf{G}}^{e e}\left(\mathbf{r}, \mathbf{x}_{\star}\right) \cdot \underline{\mathbf{M}}^{\varepsilon} \cdot \mathbf{E}_{0}^{(n)}\left(\mathbf{x}_{\star}\right)+O\left(a^{5}\right) .
$$




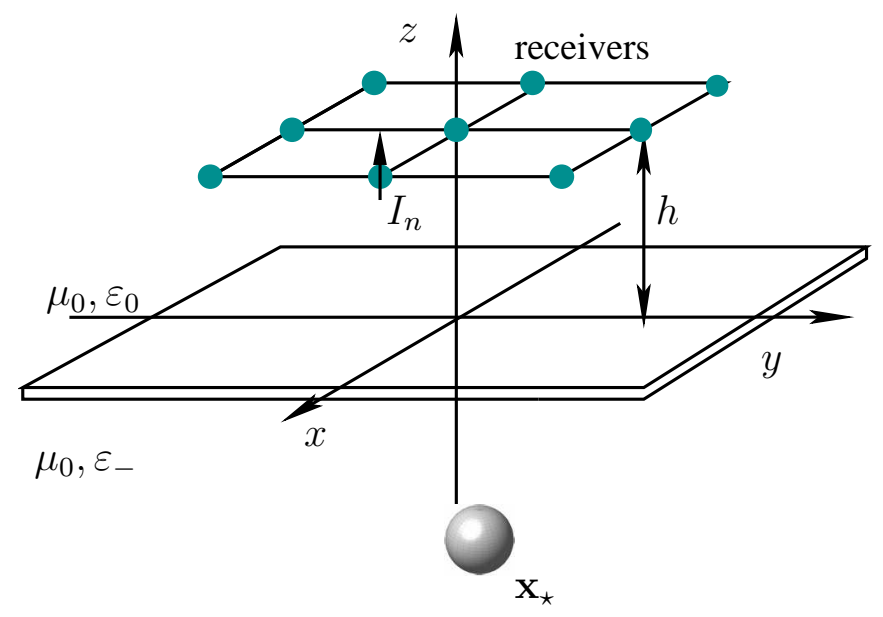

Figure 1. Configuration: two homogeneous dielectric half spaces, a homogeneous spherical dielectric inclusion buried in the lower one, a square planar array of emitting and receiving vertical electric dipoles in the upper space, symmetric with respect to the $z$-axis (each dipole is fed by a current $I_{n}$ when operated in transmission mode).

Since the inclusion lies fully within the lower half space and since receiver and source arrays are coincident in the upper one, only the transmitted part of the Green dyad [15] is needed. $\underline{\mathbf{M}}^{\varepsilon}$ is the Polarization Tensor (PT),

$$
\underline{\mathbf{M}}^{\varepsilon}=i \omega^{3}\left(\varepsilon_{\star}-\varepsilon_{-}\right) \underline{\mathbf{M}}\left(\varepsilon_{\star} / \varepsilon_{-} ; V\right)
$$

where $\underline{\mathbf{M}}\left(\varepsilon_{\star} / \varepsilon_{0} ; V\right)$ is the polarization tensor of the inclusion in the configuration.

The inclusion is buried under a planar interface, and the polarization tensor should account for it (i.e., for electromagnetic coupling between inclusion and interface, meaning here a peculiar two-body system). To calculate it, one specializes (G. Dassios, private communication [2006]) the analytical derivation of [12] carried out for two (disjoint) dielectric spheres in free space - with the help of the bispherical coordinate system [17] (refer to Appendix and Fig. 2). Indeed, the radius $a$ of the (here) lower sphere being kept finite valued (i.e., its surface being associated to a given coordinate surface $\zeta_{1}<0$ ), if the radius of (here) the upper sphere tends to infinity, the latter simply sweeps the half space $z>0$ (i.e., the interface $z=0$ is such that $\zeta=0$ ).

The polarization tensor of a body at finite non-zero distance from the interface follows from [13] as

$$
\underline{\mathbf{M}}\left(\varepsilon_{\star} / \varepsilon_{-} ; V\right)=\left(\frac{\varepsilon_{\star}}{\varepsilon_{-}}-1\right) \int_{s} \hat{\nu} \mathbf{u}^{-}\left(\mathbf{r}, \varepsilon_{\star} / \varepsilon_{-}\right) d s(\mathbf{r})+|V| \underline{\mathbf{I}},
$$

where the integration is performed on the inclusion surface with outgoing normal $\hat{\nu}$. The integral involves a threecomponent harmonic function (potential) $\mathbf{u}^{-}$within the sphere which is satisfying the following transmission 


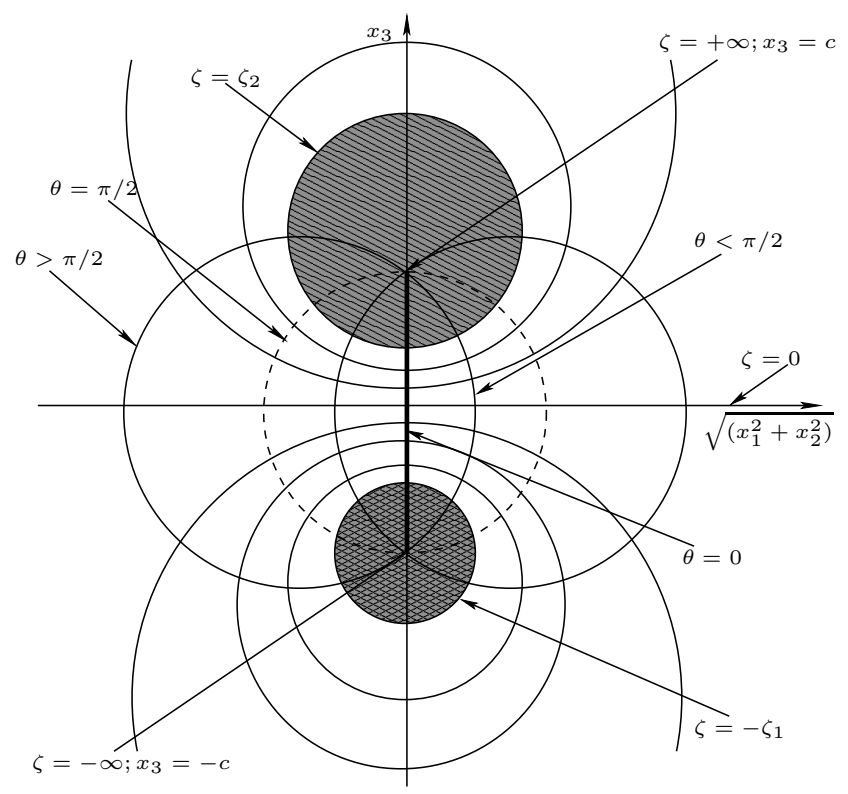

FiguRE 2. The bispherical coordinate system, and its connection to the Cartesian coordinate system. Sphere centers lie on the $x_{3}$-axis, sphere 1 at $x_{3}<0$, sphere 2 at $x_{3}>0$. Lines of constant $\theta$ pass through the focal points; when $\theta<\pi / 2$ the coordinate surfaces look like lemons, and like apples at $\theta>\pi / 2$. Surfaces of spheres 1 and 2 are $-\zeta_{1}$ and $\zeta_{2}$. $\phi$ is the rotation angle around the $x_{3}$-axis.

problems for the Laplace equation ( + conditions of cancellation at infinity):

$$
\left\{\begin{array}{l}
\Delta u_{i}^{-}=0 \\
\Delta u_{i}^{=}=0 \\
\Delta u_{i}^{+}=0 \\
u_{i}^{-}=u_{i}^{=}, \quad \zeta=-\zeta_{1} \\
\frac{\partial}{\partial \nu} u_{i}^{-}=\gamma \frac{\partial}{\partial \nu} u_{i}^{=}+\hat{\nu} \cdot \hat{x}_{i}, \quad \zeta=-\zeta_{1}, \\
u_{i}^{-}=u_{i}^{+}, \quad \zeta_{2}=0, \\
\frac{\partial}{\partial \nu} u_{i}^{-}=\beta \frac{\partial}{\partial \nu} u_{i}^{+}, \quad \zeta_{2}=0 \\
u_{i}^{+}=o\left(\frac{1}{r^{2}}\right), \quad r \rightarrow \infty
\end{array}\right.
$$

In the above, $\mathbf{u}^{=}$is the potential inside the sphere, $\mathbf{u}^{-}$is the one outside the sphere in the lower half space, and $\mathbf{u}^{+}$is the one in the upper half space; these potentials are expressed for $i=1,2,3$, successively. $\beta=\varepsilon_{0} / \epsilon_{-}$ and $\gamma=\varepsilon_{\star} / \epsilon_{-}$are the dielectric contrasts between upper and lower half spaces and the one between sphere and lower half space. In particular, using the material in Appendix on the $R$-separability of the Laplace equation $(R(\zeta, \theta)=\cosh \zeta-\cos \theta)$, one proves that $\mathbf{u}^{-}(\mathbf{r}, \gamma)=\left(u_{1}^{-}, u_{2}^{-}, u_{3}^{-}\right)$for $-\zeta_{1}<\zeta<0$ is given in exact series form 
as

$$
\begin{aligned}
u_{1}^{-} & =\sqrt{R(\zeta, \theta)} \sum_{n=1}^{+\infty}\left(C_{n}^{(1)} e^{\left(n+\frac{1}{2}\right) \zeta}+D_{n}^{(1)} e^{-\left(n+\frac{1}{2}\right) \zeta}\right) P_{n}^{1}(\cos \theta) \cos \phi \\
u_{2}^{-} & =\sqrt{R(\zeta, \theta)} \sum_{n=1}^{+\infty}\left(C_{n}^{(2)} e^{\left(n+\frac{1}{2}\right) \zeta}+D_{n}^{(2)} e^{-\left(n+\frac{1}{2}\right) \zeta}\right) P_{n}^{1}(\cos \theta) \sin \phi \\
u_{3}^{-} & =\sqrt{R(\zeta, \theta)} \sum_{n=0}^{+\infty}\left(C_{n}^{(3)} e^{\left(n+\frac{1}{2}\right) \zeta}+D_{n}^{(3)} e^{-\left(n+\frac{1}{2}\right) \zeta}\right) P_{n}(\cos \theta)
\end{aligned}
$$

where coefficients $C_{n}^{(i)}$ and $D_{n}^{(i)}$ are derived in practice as the solution of an infinite linear system via a cut-off method, e.g., [12]. This provides the polarization tensor $\underline{\mathbf{M}}\left(\varepsilon_{\star} / \varepsilon_{-} ; V\right)$ after integration. It is diagonal with two independent components $M_{11}=M_{22}$ (parallel to the interface), and $M_{33}$ (perpendicular to it):

$$
\begin{aligned}
M_{11}(\gamma ; V)= & M_{22}(\gamma ; V)=(\gamma-1) \pi c^{2} \sinh \zeta_{1} \int_{0}^{\pi} \frac{\sin \theta^{2}}{R(\zeta, \theta)^{\frac{5}{2}}} \\
& \times \sum_{n=1}^{+\infty}\left(C_{n}^{(1)} e^{-\left(n+\frac{1}{2}\right) \zeta_{1}}+D_{n}^{(1)} e^{\left(n+\frac{1}{2}\right) \zeta_{1}}\right) P_{n}^{1}(\cos \theta) d \theta+|V|, \\
M_{33}(\gamma ; V)= & (\gamma-1) 2 \pi c^{2} \sinh \zeta_{1} \int_{0}^{\pi} \frac{\sin \theta}{R(\zeta, \theta)^{\frac{5}{2}}}\left[-\sinh \zeta_{1}+R\left(\zeta_{1}, \theta\right) \operatorname{coth} \zeta_{1}\right] \\
& \times \sum_{n=0}^{+\infty}\left(C_{n}^{(3)} e^{-\left(n+\frac{1}{2}\right) \zeta_{1}}+D_{n}^{(3)} e^{\left(n+\frac{1}{2}\right) \zeta_{1}}\right) P_{n}^{0}(\cos \theta) d \theta+|V|
\end{aligned}
$$

where $P_{n}^{m}$ is the Legendre polynomial of degree $n$ and order $m=0,1, \cdots, n$ and $c$ is the half focal distance in the bispherical system (see Appendix). The components are computed in practice by replacing every $\sum_{n=0 / 1}^{+\infty}$ by $\sum_{n=0 / 1}^{N}, N$ being increased until satisfactory convergence [12]. If coupling between the inclusion and the interface can be ignored, which is expected to hold true when the inclusion is far enough from the interface (say, beyond about $3 a$ ), the polarization tensor reduces to the constant one of a single (isolated) sphere in free space, i.e., $\underline{\mathbf{M}}\left(\varepsilon_{\star} / \varepsilon_{-} ; V\right)=\frac{3 \varepsilon_{-}}{2 \varepsilon_{-}+\varepsilon_{\star}}|V| \underline{\mathbf{I}}$.

\subsection{The full-field method (CDM)}

The Coupled Dipole Method (CDM), see reference in the Introduction, is also based on the LippmannSchwinger domain integral formulation. Now, the scatterer under study is discretized into a set of $L$ subunits arranged on a cubic lattice. If the size of the subunit is small enough compared to the wavelength of the illumination, it can be assumed that the field is uniform over one subunit. Hence, the field at each subunit can be written, in the case at hand, as

$$
\mathbf{E}^{(n)}\left(\mathbf{r}_{i}\right)=\mathbf{E}_{0}^{(n)}\left(\mathbf{r}_{i}\right)+\sum_{j \neq i}^{L} \omega^{2} \mu_{0} \varepsilon_{-} \underline{\mathbf{G}}^{e e}\left(\mathbf{r}_{i}, \mathbf{r}_{j}\right) \alpha_{j} \mathbf{E}^{(n)}\left(\mathbf{r}_{j}\right)
$$

where $\alpha_{j}=3 \frac{\varepsilon_{\star}-\varepsilon_{-}}{\varepsilon_{\star}+2 \varepsilon_{-}} V_{j}$ is the associated polarizability, and $V_{j}$ is the volume of each cubic element. The field at each subunit is obtained by solving the linear system represented by Eq. (2.8). So, the scattered field can be computed from

$$
\mathbf{E}_{s}^{(n)}(\mathbf{r})=\sum_{j=1}^{L} \omega^{2} \mu_{0} \varepsilon-\underline{\mathbf{G}}^{e e}\left(\mathbf{r}, \mathbf{r}_{j}\right) \alpha_{j} \mathbf{E}^{(n)}\left(\mathbf{r}_{j}\right)
$$

The exact dyadic Green function and multiple scattering are accounted for in full, i.e., the only approximation arises from the discretization, which must be fine enough to capture accurately the field behavior (which also means that the boundaries of the scatterer at hand be well reproduced) without leading to excessive computation time in practice. 


\section{The NON-ITERATIVE IMAGING}

Here, we sketch the approach, keeping in background the ample theoretical material available in references in the Introduction. We assume coincident source-receiver arrays of $M$ dipoles with fixed amplitudes (for simplicity) $I_{n}=I_{0}$. For any space point $\mathbf{x}$, the matrix $G^{e}(\mathbf{x})$ of size $M \times 3$ reads as

$$
G^{e}(\mathbf{x})=\left[\underline{\mathbf{G}}^{e e, T}\left(\mathbf{x}, \mathbf{r}_{1}\right) \cdot \hat{z}, \ldots, \underline{\mathbf{G}}^{e e, T}\left(\mathbf{x}, \mathbf{r}_{M}\right) \cdot \hat{z}\right]^{t}
$$

where $\mathbf{r}_{p}, p=1, \ldots, M$, is the position of the $p$ th array element and $T$ denotes the transmitted part (source and observation are separated by the interface) of the Green dyad. Using (2.2), the MSR matrix $A$ of size $M \times M$ is formed, and decomposed as

$$
A=G\left(\mathbf{x}_{\star}\right) \underline{\mathbf{M}}^{\varepsilon} G^{t}\left(\mathbf{x}_{\star}\right), G(\mathbf{x})=\left[G^{e}\right](\mathbf{x}) .
$$

If the dimension of the signal space, $s$, is known beforehand or if one is able to estimate it from the singular value decomposition $A=U \Sigma V^{*}$ of $A$ (say, $s$ significant singular values emerge from the $M$ ones, the remaining ones being of much lower magnitude, equal to zero if the MSR matrix was calculated indeed by the asymptotic method), then the MUSIC (MUltiple SIgnal Classification) algorithm readily applies. In particular, for the configuration depicted in Fig. 1, it can be shown that $s=3$ in the case of a single dielectric inclusion - this is under the assumption that the inclusion is small enough with respect to the wavelength in the embedding medium, refer to [14] for a numerical illustration of the increase of $s$ whenever a spherical scatterer is inflated and consequences in terms of imaging. Thus, for any vector $e \in \mathbb{C}^{3}$, such as $G(\mathbf{x}) \cdot e \neq 0$, and for any space point $\mathbf{x}$ in a prescribed search box, a map of the estimator $W(\mathbf{x})$ as the inverse of the squared Euclidean distance from the Green vector $G(\mathbf{x}) \cdot e$ to the signal space

$$
W(\mathbf{x})=1 / \sum_{i=s+1}^{M}\left|\left\langle U_{i}, G(\mathbf{x}) \cdot e\right\rangle\right|^{2},
$$

peaks (to infinity, in theory) at the center of the inclusion $\mathbf{x}_{\star}$, the calculation of $W(\mathbf{x})$ being performed in practice at $l \times l \times l$ nodes which should finely (with respect to the wavelength again) span the search box. At this stage, we should emphasize that the computation time of the transmitted part of the Green dyad could become prohibitive since we have to calculate for all such nodes its value at each receiver (they are $M$ of them). The Leading Order Approximation as proposed in [11] then enables us to reduce the computation time of $W$ while (under proper conditions as illustrated next) keeping a suitable accuracy.

\section{Numerical Simulations}

In the simulations, the spherical dielectric inclusion is of radius $a=0.05 \mathrm{~m}$ and permittivity $\varepsilon_{\star}=12 \varepsilon_{0}$. The half space of burial is of permittivity $\varepsilon_{-}=4 \varepsilon_{0}$. The operation frequency is set to $300 \mathrm{MHz}$, wavelengths in the upper and lower half spaces then being $\lambda_{+}=1$ and $\lambda_{-}=0.5$, respectively, all dimensions from now being given in meters. We consider that the inclusion center is shifted with respect to the symmetry axis of the array, at $x_{\star}=0.15$ and $y_{\star}=0.23$, its depth being $z_{\star}=-d, d=1,0.2,0.1$.

\subsection{Influence of coupling on polarization tensor and scattered field}

The diagonal elements of the polarization tensor taking in account coupling to the interface and calculated via the bispherical system and those of the one in free space are compared in Fig. 3 as a function of the ratio $d / a$. As soon as the depth of burial $d$ is larger than about $3 a$, the tensors are identical in numerical practice, and the sphere behaves as if it was isolated in free space. When the sphere gets closer and closer to the interface without contact (if contact, the modeling breaks down, and the tensor should be estimated in a different coordinate system), the coefficients separate more and more. Yet, the discrepancy with respect to the isolated sphere remains small (less than 5\%) while coefficients $M_{11}=M_{22}$ and $M_{33}$ vary similarly and differ from one another 


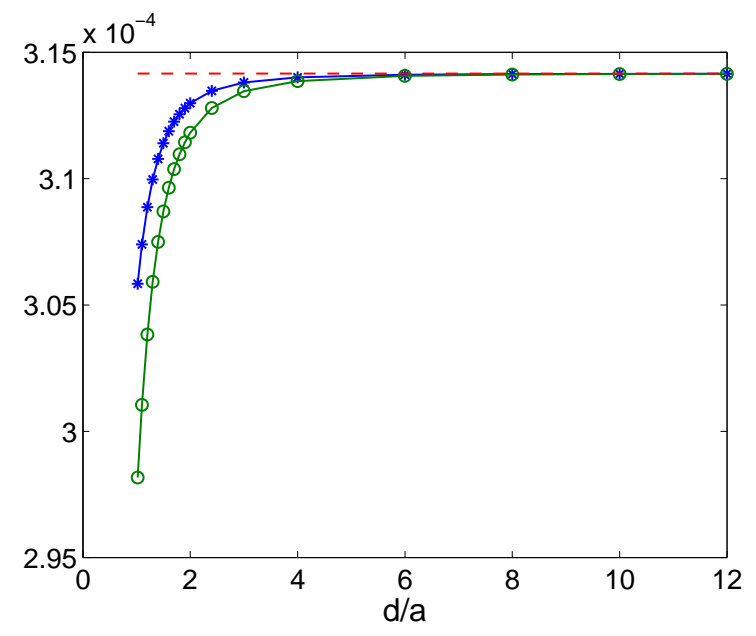

Figure 3. The diagonal elements of the polarization tensor matrix $M_{11}$ and $M_{22}\left(\left(^{*}\right)\right.$ curve), $M_{33}$ ( (o) curve) computed in the bispherical system vs. depth-to-size ratio $d / a$, are compared to the common value of $M$ (dash curve) for an isolated sphere in free space.
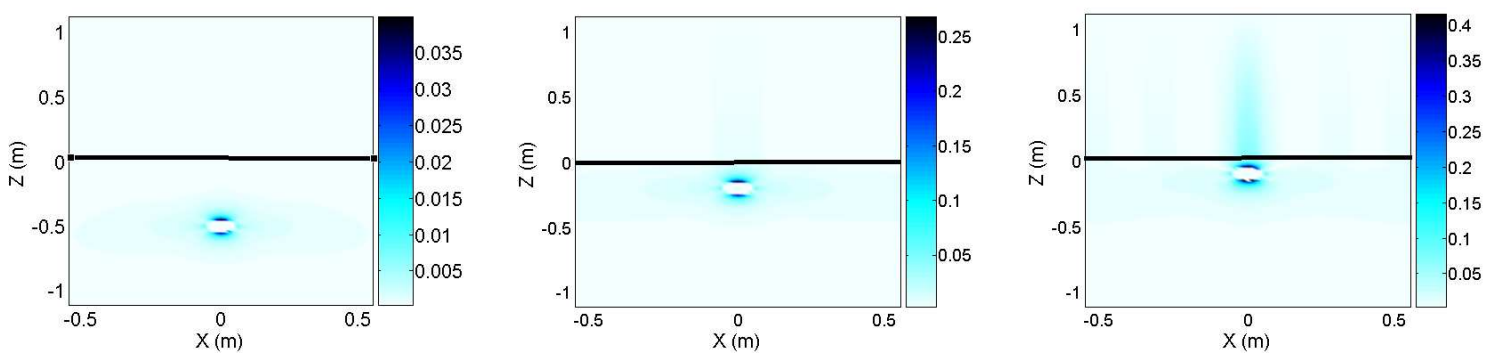

FiguRE 4. The magnitude of the differences between the vertical components of the scattered field obtained via the asymptotic formula with coupling (the polarization tensor is the true one) and without coupling (the polarization tensor is the one of an isolated sphere) within the vertical $(x, z>0)$-plane for various depths $d$ : Left $d=0.5$; middle $=0.2 ;$ right $=0.1$. The values of $N$ providing numerical convergence in the two-body system are $9,16,25$ for $d=0.5,0.2,0.1$, respectively.

by far less. Similar conclusions could be drawn for other dielectric contrasts of the sphere vs. the lower half space.

Setting one vertical dipole transmitter at location $(-1.75,1.75,0.5)$, and computing in the plane $(x, z>0)$, for different depths $d$, the difference of magnitude between the vertical $(z)$ components of the scattered field obtained via the asymptotic formula with coupling (involving the true PT) and without coupling (using the PT of an isolated sphere) is illustrated in Fig. 4, taking as reference the wavelength $\lambda_{+}$in the upper medium. The influence of coupling appears strong whenever the field is observed close to the interface, say, at about $0.2 \lambda_{+}$ and below. When the sphere is close to the interface at, as an average, $\lambda_{-}$or less, this effect is emphasized.

\subsection{Fields and images from asymptotic method and CDM}

As previously, a vertical dipole transmitter is at location $(-1.75,1.75,0.5)$. We look at the $E_{z}$ components of the scattered field at the position of a $8 \times 8$ dipole array, the array spacing being $\lambda_{+} / 2$, the array height 

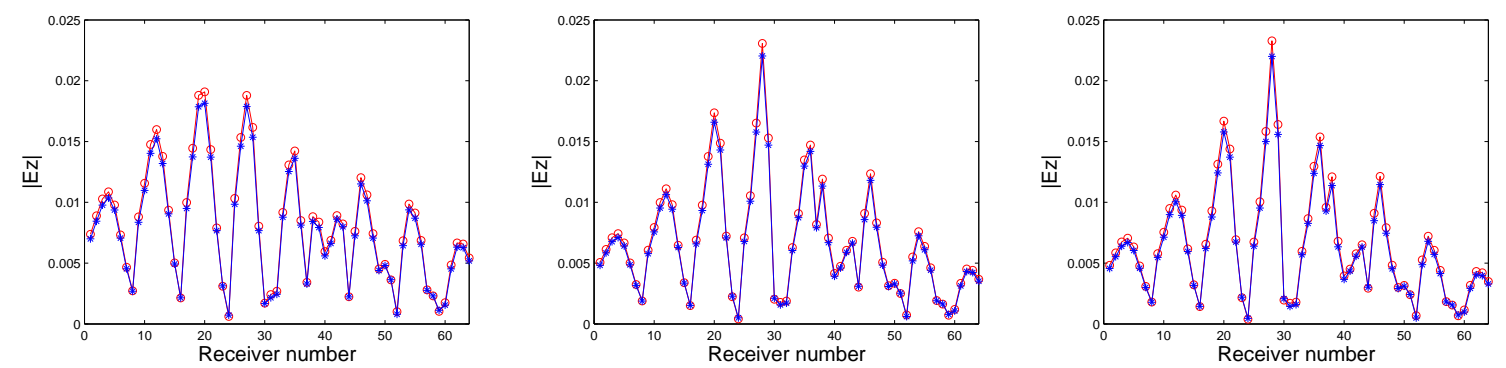

Figure 5. Comparison of the amplitudes of the vertical $E_{z}$-component of the scattered field computed by the asymptotic method with full polarization tensor (o) curve) and by CDM $((*)$ curve) for several $d$ when the $(-1.75,1.75,0.5)$-dipole is radiating: Left $d=1$; middle $=0.2$; right $=0.1$. The receivers are numbered from 1 to 64 , by sets of 8 , one array line after another.

being $h=\lambda_{+} / 2$. In Fig. 5, we show a comparison of this scattered field computed according to the asymptotic method and to CDM, for several depths $d$. With CDM, the sphere is discretized into 4224 cubical elements in order to get both an accurate representation of its boundary and of the field within it, while maintaining acceptable CPU time overall. The agreement appears excellent, being emphasized again that the sphere radius is small in absolute value yet not so small if we take as reference the wavelength $\lambda_{-}\left(a=\lambda_{-} / 10\right)$, whereas its dielectric permittivity of 12 compared to 4 in the lower half space means its electrical size is in effect 1.7 times larger. Let us notice that the full polarization tensor is used here, not the one of an isolated sphere - yet, this would have no visible effects down to about $d=0.15(=3 a)$.

Let us now assume that the MSR matrix is calculated for the $M$ array either via the asymptotic method or via CDM. After singular value decomposition, the MUSIC algorithm is applied in order to locate the position of the sphere (within a box centered on the $z$-axis with $21 \times 21 \times 21$ nodes, $\lambda_{-} / 10$ stepped). Fig. 6 shows what happens from CDM data when the Green dyad is exact or is calculated via the LOA - the latter reduces the computation time by a factor of more than 50 with respect to an exact calculation in the imaging procedure. The quality of the imaging results is not the same, the LOA significantly blurring the image into the longitudinal direction. But we have assumed here that the data are noiseless, which is not so realistic an assumption, and that we pick up the three good ones, which is not so straightforward a task in view of the slowly-decaying spectrum of the MSR matrix.

To estimate the proper number of significant singular values from the CDM data is a challenge per se, as shown in Fig. 6. If we use the asymptotic method to calculate the MSR matrix, the three expected singular values are easy to discriminate from all others, which are close to zero save numerical noise. But, in real world, we cannot reach that accurate a set of data, neither assume that asymptotic models so closely provide the data, and noise is inevitable. The latter has a rather dramatic effect on the singular spectrum, as it shows when we add a noise of $10 \mathrm{~dB}$ SNR onto the MSR matrix. Indeed, now, we efficiently distinguish the signal subspace from the noise subspace with CDM data, without affecting the discrimination with asymptotic data, as is exhibited in following figures. Noise being input, at $d=1$, i.e., $2 \lambda_{-}$(Figs. 7 and 8 ) (deep inclusion), or at $d=0.2$, i.e., $0.4 \lambda_{-}$(Fig. 9) (shallow one), good locations are found, with isosurface at $80 \%$ of the maximum value of the MUSIC cost functional. Let us emphasize that the resolution is non-isotropic, as is expected from the type of configuration involving a source/receiver array which is fixed at some distance above the scatterer.

\section{CONCLUSiOn}

Asymptotic formulas of the field scattered by a small spherical inclusion buried in a half space yield fields which are closely resembling to those computed by the full-field CDM method, even at very shallow depths, for a small inclusion as considered herein. This involves the calculation of the polarization tensor in a bispherical coordinate system in order to approach the coupling between inclusion and interface. The imaging itself appears 

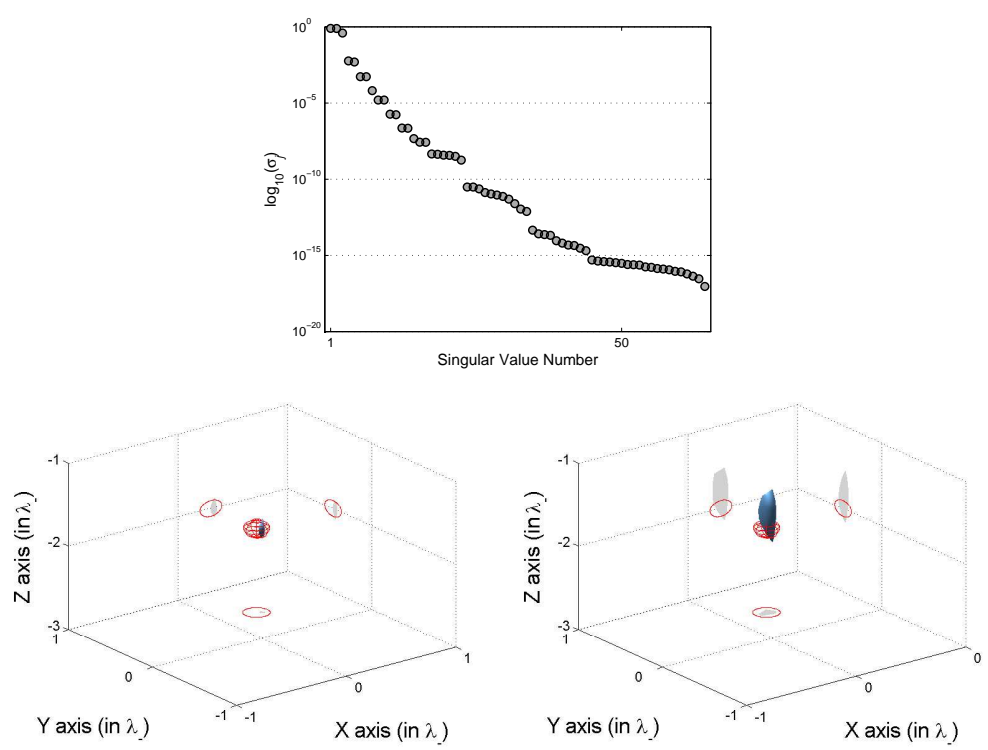

FigURE 6. MUSIC imaging (isosurface at $80 \%$ ) of the sphere buried at $d=1$ using the 3 singular values of highest amplitude of the MSR matrix computed by the CDM. Top: singular spectrum; bottom left: subwavelength-resolved image obtained via the Green dyad calculated by the exact formulation; bottom right: longitudinally-blurred image obtained via the Green dyad calculated by the LOA.

rather robust, be it carried out from asymptotic or exact MSR matrices, and to a lesser degree when speeding up the computation of the Green dyad by a first-order approximation (LOA). The numerical simulations help us to appraise super-resolution, or better said, super-localization, since we aim at the location of a single sphere, and separation between two spheres is not envisaged.

For noisy data, when the first three singular values associated to the signal subspace emerge well enough from those of the noise subspace and can be picked up properly, localization is almost the same for a sphere centered at some distance $\left(2 \lambda_{-}\right)$from the interface and for one centered at short distance $\left(2 \lambda_{-} / 5\right)$. It is always better in the transverse directions than in the longitudinal one in view of the fixed array position. A full-wave (CDM) MSR matrix and the asymptotic one yield the same results. But, whether we use the exact Green dyad in the imaging (at high computational cost) or the LOA one (at much lesser cost), this has a significant impact on the results. Excellent transverse and longitudinal localizations (about $\lambda_{-} / 20$ and $2 \lambda_{-} / 10$, resp.) are obtained with the exact form, those deteriorating (about $\lambda_{-} / 10$ and $8 \lambda_{-} / 10$, resp.) with the LOA.

Present work devoted to two spheres in free space, using CDM and asymptotic data, should enable us to further investigate the consequences of coupling onto the imaging, and super-resolution thereof, with a less complicated Green dyad. 

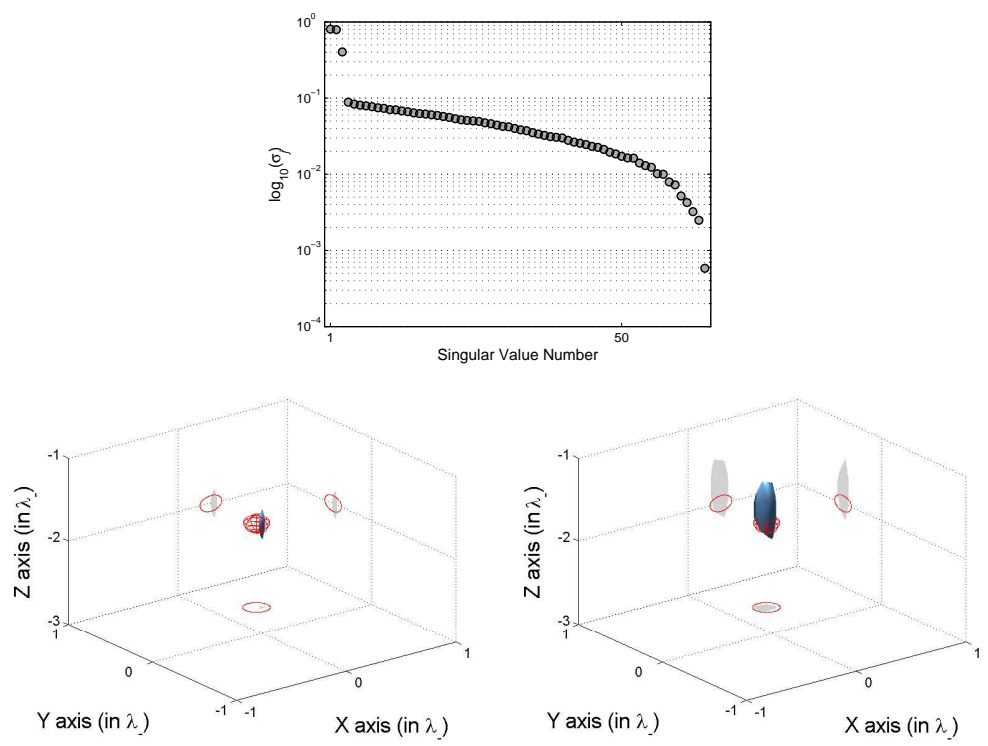

FIgURE 7. MUSIC imaging (isosurface at $80 \%$ ) of the sphere buried at $d=1$ using the 3 singular values of highest amplitude of the MSR matrix computed by the CDM, with 10 $\mathrm{dB}$ added noise. Top: singular spectrum; bottom left: image obtained via the Green dyad computed by the exact formulation; bottom right: image obtained via the Green dyad computed by the LOA.
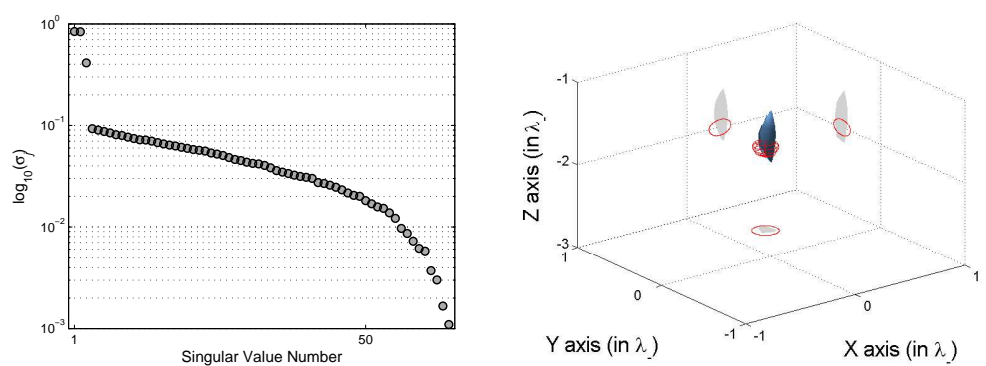

Figure 8. Same as in Fig. 7 with the MSR matrix now built from asymptotic data, with 10 $\mathrm{dB}$ added noise. Only the results with the LOA are shown.

\section{A. BisphericAl SYSTEM AND $R$-SEPARABILITY}

Let us refer to Fig. 2. The (axially symmetric) bispherical coordinate system $(\zeta, \theta, \phi)$ is connected to the Cartesian coordinate system $\left(x_{1}, x_{2}, x_{3}\right)$ :

$$
\left\{\begin{aligned}
x_{1} & =\frac{c}{R(\zeta, \theta)} \sin \theta \cos \phi, & & \\
x_{2} & =\frac{c}{R(\zeta, \theta)} \sin \theta \sin \phi, & 0 & \leq \infty \leq+\infty \\
x_{3} & =\frac{c}{R(\zeta, \theta)} \sinh \zeta, & 0 & \leq \phi<2 \pi
\end{aligned}\right.
$$



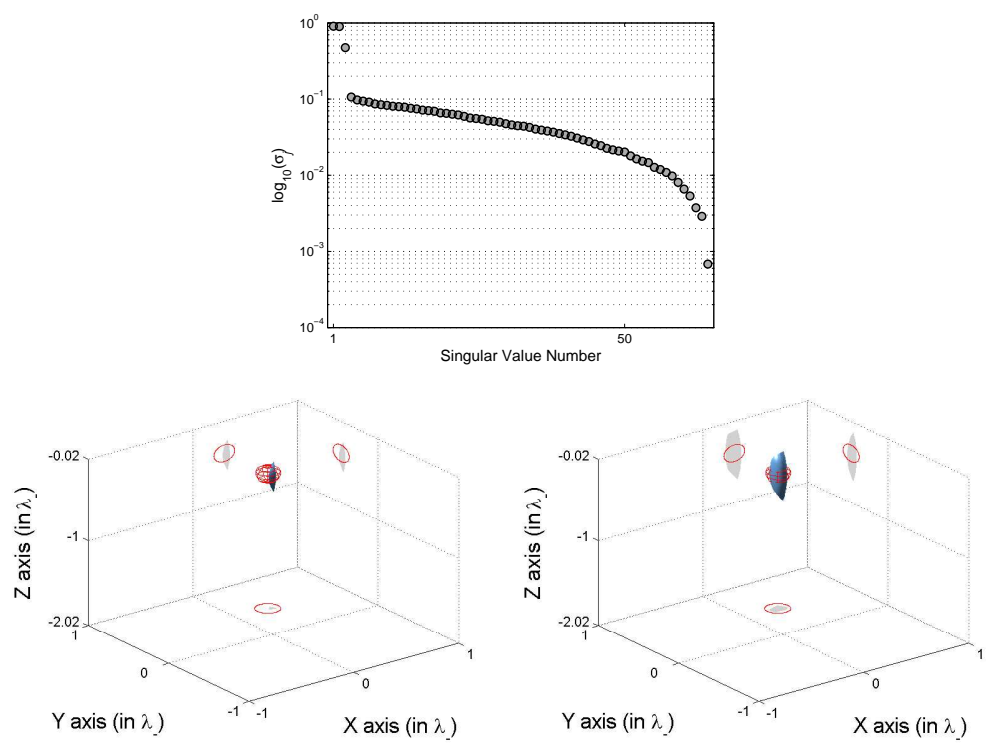

Figure 9. Same as in Fig. 7 for $d=0.2$.

Coefficient $R(\zeta, \theta)=\cosh \zeta-\cos \theta ; 2 c$ is the distance between focii determined from the radii of the spheres (not in contact) and their distance center to center. Surfaces of constant $\zeta=-\zeta_{1}$ and $\zeta=\zeta_{2}$ are spheres of radii $a_{j}=c /|\sinh \zeta|$ where $j=1,2$, centered at locations $x_{1}=0, x_{2}=0$ and $x_{3}=c \operatorname{coth} \zeta$. For a single sphere (surface $\left.\zeta=-\zeta_{1}\right)$ with radius $a$ centered at $\left(d=c \operatorname{coth} \zeta_{1}\right)$ under a planar interface (coordinate surface $\zeta=0$ ), $c^{2}=d^{2}-a^{2}$. The infinitesimal surface element is $d s(r)=c^{2} \frac{\sin \theta}{R^{2}(\zeta, \theta)} d \theta d \phi$. The Laplace equation reads as

$$
\Delta u(\zeta, \theta, \phi)=\frac{R^{3}(\zeta, \theta)}{c^{2} \sin \theta}\left[\sin \theta \frac{\partial}{\partial \zeta}\left(\frac{1}{R(\zeta, \theta)} \frac{\partial}{\partial \zeta}\right)+\frac{\partial}{\partial \theta}\left(\frac{\sin \theta}{R(\zeta, \theta)} \frac{\partial}{\partial \theta}\right)+\frac{R^{2}(\zeta, \theta)}{c^{2} \sin ^{2} \theta} \frac{\partial^{2}}{\partial^{2} \phi}\right]
$$

Any harmonic function $u$ satisfies so-called $R$-separation, and $R$-eigenfunctions regular at the origin are of the form

$$
u=\sqrt{R(\zeta, \theta)}\left\{\begin{array}{c}
e^{\left(n+\frac{1}{2}\right) \zeta} \\
e^{-\left(n+\frac{1}{2}\right) \zeta}
\end{array}\right\} P_{n}^{m}(\cos \theta)\left\{\begin{array}{c}
\cos m \phi \\
\sin m \phi
\end{array}\right\} .
$$

In addition to textbook [17], refer to [7] and [12] for further details about how to exploit the bispherical system for solving in analytical fashion scattering problems for two spheres (see also the exact approach by [8]).

\section{REFERENCES}

[1] H. Ammari and H. Kang, Reconstruction of Small Inhomogeneities from Boundary Measurements Springer, Berlin, 2004.

[2] H. Ammari and H. Kang, Polarization and Moment Tensors with Applications in Inverse Problems and Effective Medium Theory Springer, New York, 2007.

[3] H. Ammari, E. Iakovleva, and H. Kang, Reconstruction of a small inclusion in a two-dimensional open waveguide, SIAM J. Appl. Math., vol. 65, pp; 2107-2127, 2005.

[4] H. Ammari, E. Iakovleva, and D. Lesselier, A MUSIC algorithm for locating small inclusions buried in a half-space from the scattering amplitude at a fixed frequency, Multiscale Modeling Simulation, vol. 3, pp. 597-628, 2005.

[5] H. Ammari, E. Iakovleva, D. Lesselier, and G. Perrusson, MUSIC-type electromagnetic imaging of a collection of small threedimensional bounded inclusions, SIAM J. Scientific Comput., vol. 29, pp. 674-709, 2007.

[6] H. Ammari, H. Kang, E. Kim, and M. Lim, Reconstruction of closely spaced small inclusions, SIAM J. Num. Anal., vol. 42, pp. 2408-2428, 2005. 
[7] A. Charalambopoulos, G. Dassios, M. Hadjinicolaou, An analytic solution for low-frequency scattering by soft spheres, SIAM J. Appl. Math., vol. 58, pp. 370-386, 1998.

[8] P. C. Chaumet, and J.-P. Dufour, Electric potential and field between two different spheres, J. Electrostatics, vol. 43, pp. $145-159,1998$.

[9] P. C. Chaumet, A. Sentenac, and A. Rahmani, Coupled dipole method for scatterers with large permittivity, Physical Rev. E, vol. 70, 036606, 2004.

[10] T. J. Cui and W. C. Chew, Efficient evaluation of Sommerfeld integrals for TM wave scattering by buried objects, J. Electromagn. Waves Appl., vol. 12, pp. 607-657, 1998.

[11] T. L. Cui and W. C. Chew, Fast evaluation of Sommerfeld integrals for electromagnetic scattering and radiation by threedimensional buried objects, IEEE Trans. Geosci. Remote Sens., vol. 37, pp. 887-900, 1999.

[12] G. Dassios, M. Hadjinicolaou, G. Kamvyssas, and A. N. Kandili, On the polarizability potential for two spheres, Int. J. Engineer. Science, vol. 44, pp. 1520-1533, 2006.

[13] G. Dassios and R. Kleinman, Low Frequency Scattering, Clarendon Press, Oxford, 2000.

[14] S. Gdoura, D. Lesselier, G. Perrusson, and P. C. Chaumet, MUSIC-type imaging of dielectric spheres from single-frequency, asymptotic and exact array data, pp. 348-352, Proc. Progress In Electromagnetic Research Symposium (PIERS'2007), Kong, J. A. ed., Prague, 2007.

[15] E. Iakovleva, S. Gdoura, D. Lesselier, and G. Perrusson, Multi-static response matrix of a 3-D inclusion in half space and MUSIC imaging, IEEE Trans. Antennas Propagat., vol. 55 , pp. 2598-2609, 2007.

[16] E. Iakovleva and D. Lesselier, Multi-Static Response matrix of spherical scatterers and the back-propagation of singular fields, IEEE Trans. Antennas Propagat., vol. 56, pp. 825-833, 2008.

[17] P. Moon and D. Spencer, Eleven Co-ordinate Systems, Field Theory Handbook, Springer, Berlin, 1961.

[18] Y. Zhong and X. Chen, MUSIC imaging and electromagnetic inverse scattering of multiply scattering small anisotropic spheres, IEEE Trans. Antennas Propagat., vol. 55, pp. 3342-3549, 2007. 